\title{
The Endocannabinoid, 2-Arachidonoyl Glycerol, Induces Growth Cone Collapse and Neurite Retraction in Growing Peripheral Sensory Neurons
}

\author{
Chizu Aso1, Tomonori Takazawa2 ${ }^{2}$, Tatsuo Horiuchi², Shigeru Saito ${ }^{2}$ \\ ${ }^{1}$ Department of Anesthesiology, National Hospital Organization Takasaki General Medical Center, Takasaki, \\ Japan \\ ${ }^{2}$ Department of Anesthesiology, Gunma University Graduate School of Medicine, Maebashi, Japan \\ Email: ${ }^{*}$ takazawt@gunma-u.ac.jp
}

Received 19 November 2014; revised 10 December 2014; accepted 17 December 2014

Academic Editor: Anamitra Ghosh, Van Andel Research Institute, USA

Copyright (C) 2015 by authors and Scientific Research Publishing Inc.

This work is licensed under the Creative Commons Attribution International License (CC BY).

http://creativecommons.org/licenses/by/4.0/

(c) (i) Open Access

\begin{abstract}
Cannabis has a detrimental impact on the developing nervous system. Therefore, regular consumption of cannabis by pregnant and lactating woman poses a potential risk to neuronal growth in fetuses and infants. Indeed, endogenous cannabis-like molecules called endocannabinoids regulate many physiological processes, including neurogenesis, axon guidance, and synaptic plasticity through CB1 receptors. To investigate the physiological role of CB1 receptors on peripheral sensory nerve growth, the endocannabinoid 2-arachidonoyl glycerol was added to cultured chick dorsal root ganglion neurons. This compound inhibited neurite elongation and induced growth cone collapse in a dose- and time-dependent manner. These data suggest that caution should be exercised regarding maternal cannabis use during pregnancy. Because ectopic sprouting and abnormal neuronal network connections are considered to be a cause of neuropathic pain, our current data imply an additional role of endocannabinoids as inhibitors of the formation of painmaintenance networks.
\end{abstract}

\section{Keywords}

Endocannabinoid, CB1 Receptors, Dorsal Root Ganglion Neurons, Growth Cone

\footnotetext{
*Corresponding author.
}

How to cite this paper: Aso, C., Takazawa, T., Horiuchi, T. and Saito, S. (2015) The Endocannabinoid, 2-Arachidonoyl Glycerol, Induces Growth Cone Collapse and Neurite Retraction in Growing Peripheral Sensory Neurons. World Journal of Neuroscience, 5, 1-6. http://dx.doi.org/10.4236/wjns.2015.51001 


\section{Introduction}

Cannabis is considered a relatively harmless recreational agent despite gathering evidence of its detrimental impact on both the adult and the developing central nervous system (CNS) [1]. This attitude may simply reflect people's recognition that the risk of cannabis is relatively low, particularly when compared to that of tobacco and alcohol [2].

Cannabis has a small molecular size and is generally lipophilic, enabling the compound to readily cross the blood-brain or other cellular (for example, placental) barriers. Thus, regular cannabis use during pregnancy may result in relatively high concentrations of active cannabinoids in the developing fetus [3]. Moreover, cannabis and its metabolites readily pass into breast milk. When cannabis is regularly consumed by breast-feeding mothers, human milk concentrations of delta-9-tetrahydrocannabinol (THC), the main component of cannabis with psychoactive properties, may be up to 8-fold higher than simultaneously measured maternal plasma concentrations [4]. The effects of cannabis on the developing fetus and infant remain uncertain, although some evidence suggests that perinatal cannabis exposure can negatively affect fetal growth [5].

CB1 receptors (CB1Rs) are widely distributed within the brain including cerebral cortex, hippocampus, basal ganglia, and amygdala, as major targets of exogenous cannabinoids such as THC. Moreover, the most abundant endocannabinoid (endogenous cannabinoid: eCB) in the CNS, 2-arachidonoyl glycerol (2-AG), also triggers a broad range of signaling events by acting on CB1Rs. The eCB system regulates many physiological processes including neurogenesis, axon guidance, and synaptic plasticity [6]-[8]. In addition to the CNS, CB1Rs are also expressed in the peripheral nervous system [9] [10]. However, few studies have demonstrated a physiological role for CB1Rs that are expressed in peripheral neurons [11]. Therefore, whether the activation of CB1Rs in developing peripheral sensory neurons affects neuronal growth and function should be determined.

Here we study dorsal root ganglion (DRG) neurons, which are peripheral sensory neurons. The afferents of DRG neurons relay sensory information that originates from the skin to the brain. Therefore, they play a critical role in nociception including inflammatory and neuropathic pain [12]. Cultured DRG neurons isolated from chick embryos can be utilized to evaluate the effects of drugs on neuronal growth during development [13]. We previously show that several local anesthetics have direct neurotoxic effects on cultured DRG neurons [14] [15]. The purpose of the present study is to elucidate the effects of cannabinoids on peripheral sensory nerve growth and regeneration by examining neurite extension and growth cone collapse in particular. For this purpose, cultured DRG neurons are exposed to 2-AG.

\section{Materials and Methods}

This study was approval by the Institutional Animal Care Committee. Chick neural tissues were isolated from day 7 embryos. To prepare peripheral neurons, DRGs were dissected from lumbar paravertebral sites. After removing the original neurites, the explants were plated on laminin-coated coverslips and cultured in F-12 medium supplemented according to Bottenstein's method [13], containing $100 \mu \mathrm{g} / \mathrm{ml}$ bovine pituitary extract, 2 $\mathrm{mM}$ glutamine, $100 \mathrm{U} / \mathrm{ml}$ penicillin, $100 \mu \mathrm{g} / \mathrm{ml}$ streptomycin, and $20 \mathrm{ng} / \mathrm{ml}$ mouse $7 \mathrm{~S}$ nerve growth factor. Cultures were maintained at $37^{\circ} \mathrm{C}$ in $5 \%$ carbon dioxide. 2-arachidonoyl glycerol (2-AG) was purchased from Sigma Co. Ltd. (10 mM stock in ethanol).

After culturing for $20 \mathrm{hr}$, DRGs were exposed to the agents. The neurite length and percent collapse were determined after exposure for 2, 6, or $24 \mathrm{hr}$. 2-AG was prepared in pre-warmed fresh culture medium and was gently added to the culture medium. The volume of the added solution was 1/100 of the total volume of the culture medium. Cell viability was determined by exposing the cells to vehicle solution for identical durations.

The tissues were fixed with 4\% paraformaldehyde in PBS (pH 7.4) containing 10\% sucrose as described previously [14] [15] and viewed with a 40× phase objective using a phase-contrast microscope (Axiovert; Zeiss, Germany). Growth cones at the periphery of explants were scored for collapse if they were not in contact or close proximity to other growth cones or neurites. Fifty growth cones were viewed and scored per coverslip. Growth cones without filopodia and lamellipodia were counted as collapsed [14] [15]. The counting was performed by a trained examiner who was blinded to the experimental protocol.

The data are expressed as the mean \pm SD of six independent measurements. Each data point for the neurite length and growth cone collapse assays was statistically analyzed with two-way analysis of variance with Bonferroni’s method using GraphPad Prism 6 (GraphPad Software Inc., La Jolla, CA). P values less than 0.05 were considered significant. 


\section{Results}

Significant neurite elongation of cultured DRG neurons was observed over time under control condition (i.e. without 2-AG). However, the endogenous CB1R agonist 2-AG induced significant inhibition of neurite elongation with growth cone collapse as shown in Figure 1. These effects were dose- and time-dependent (two-way ANOVA, Figure 2). 2-AG $(10 \mu \mathrm{M})$ blocked axonal elongation at 6 and 24 hr after exposure $(\mathrm{P}<0.0001$, Figure 2(a)). The total neurite length $24 \mathrm{hr}$ after exposure of 2-AG (10 $\mu \mathrm{M})$ was even shorter than that before exposure suggesting severe neurotoxicity. 2-AG $(10 \mu \mathrm{M})$ significantly increased the rate of growth cone collapse 2, 6, and $24 \mathrm{hr}$ after exposure $(\mathrm{P}<0.0001$, Figure 2(b)). Even low concentrations of 2-AG $(1$ and $0.1 \mu \mathrm{M})$ increased the rate of collapse in a dose-dependent manner (Figure 2(b)). The effects of 2-AG on neurite length and rate of collapse $24 \mathrm{hr}$ after exposure are summarized in Figure 3(a) and Figure 3(b), respectively.

\section{Discussion}

In the present study, we showed that 2-AG induced growth cone collapse and inhibited axonal elongation in cultured peripheral sensory neurons. These actions were dose dependent and seemed to be pharmacological. However, we cannot completely exclude the possibility that the effect of 2-AG was not through CB1Rs, because we did not test whether the action of 2-AG was reversed by a CB1R antagonist such as AM251. Whether CB1R antagonists alone have any effect on neuronal growth of developing DRG neurons is also unclear. Testing with CB1R antagonists with neither independent neurotoxic nor neuro-pharmaceutical actions may be useful for

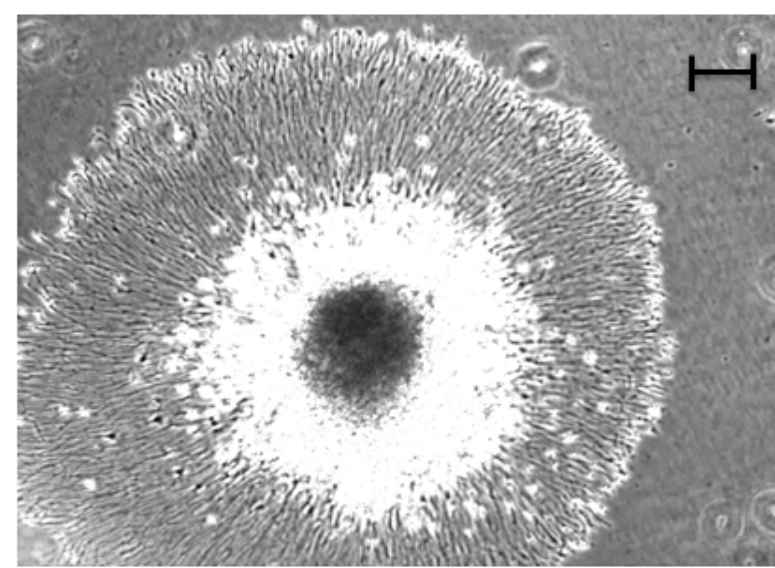

(a)

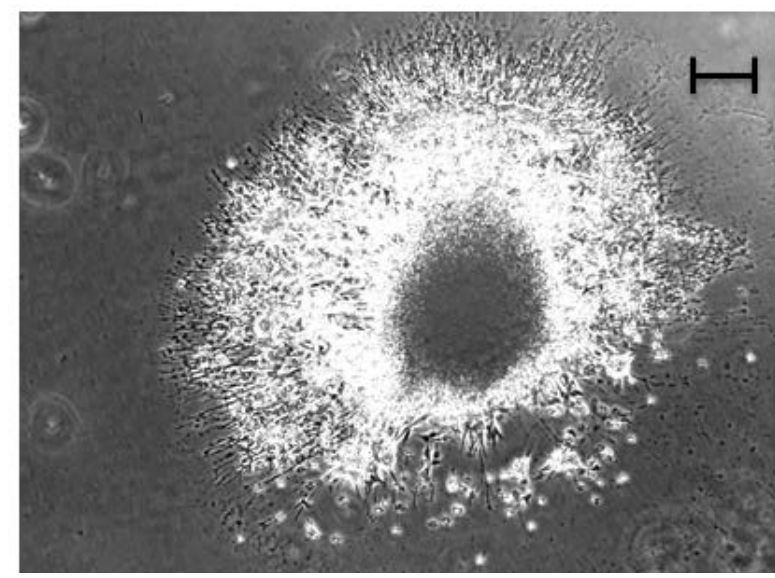

(c)

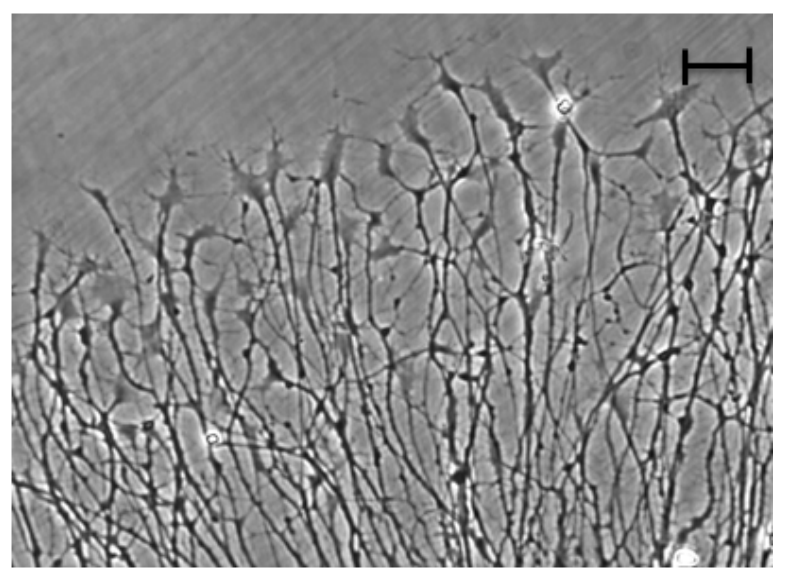

(b)

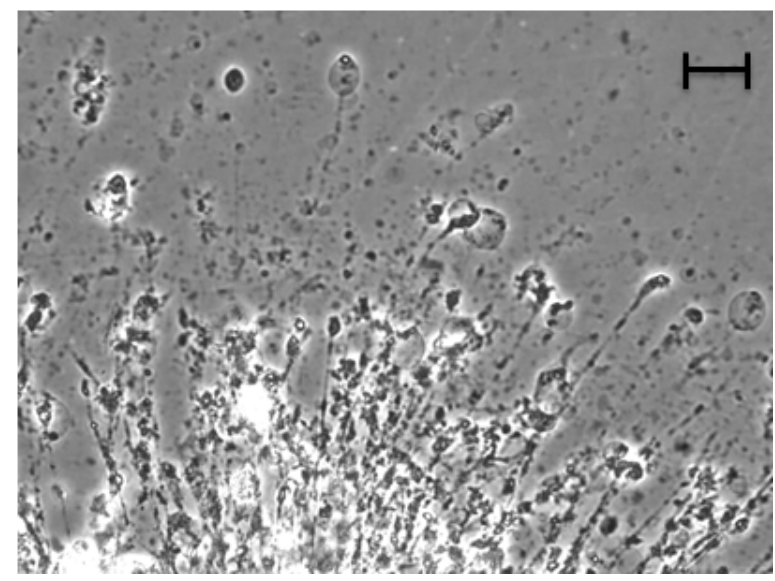

(d)

Figure 1. Typical neurite retraction and growth cone collapse induced by 2-AG in cultured DRG neurons. (a) (b) The neuron before the application of 2-AG (preexposure); (c) (d) The neuron after exposure to $10 \mu \mathrm{M} 2-\mathrm{AG}$ for $24 \mathrm{hr}$. Scale bars in (a) and (c) $=10 \mu \mathrm{m}$; bars in (b) and (d) $=60 \mu \mathrm{m}$. 


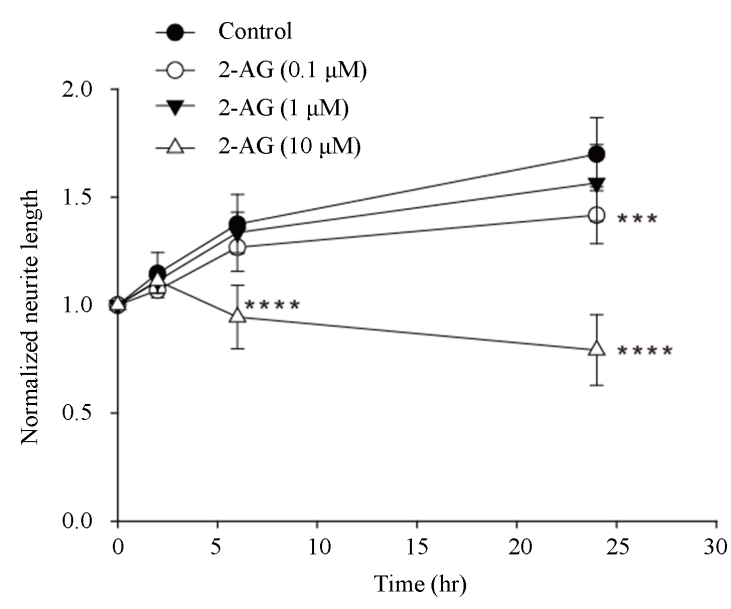

(a)

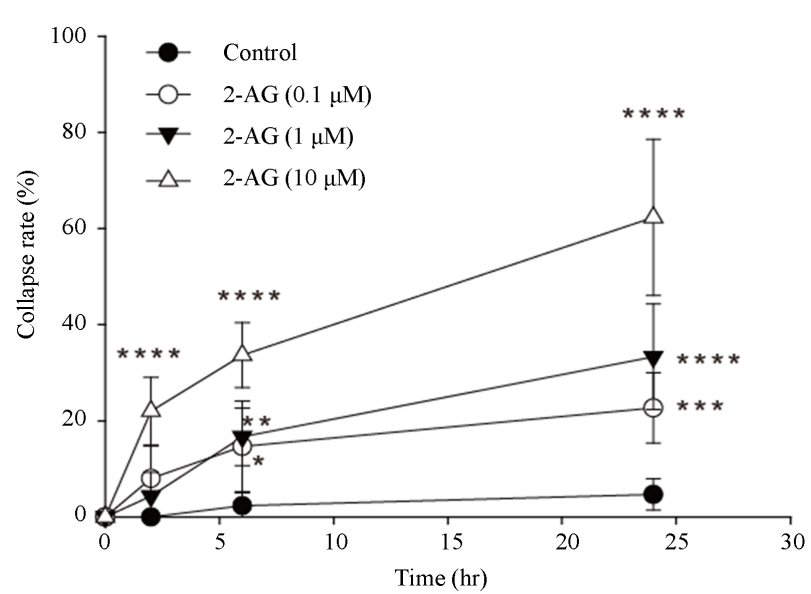

(b)

Figure 2. The effect of 2-AG on neurite length (a) and collapse rate of cultured DRG neurons. ${ }^{*} \mathrm{P}<0.05,{ }^{* *} \mathrm{P}<0.01,{ }^{* * *} \mathrm{P}<$ $0.001,{ }^{* * * *} \mathrm{P}<0.0001$, two-way ANOVA with a post-hoc Bonferroni test.

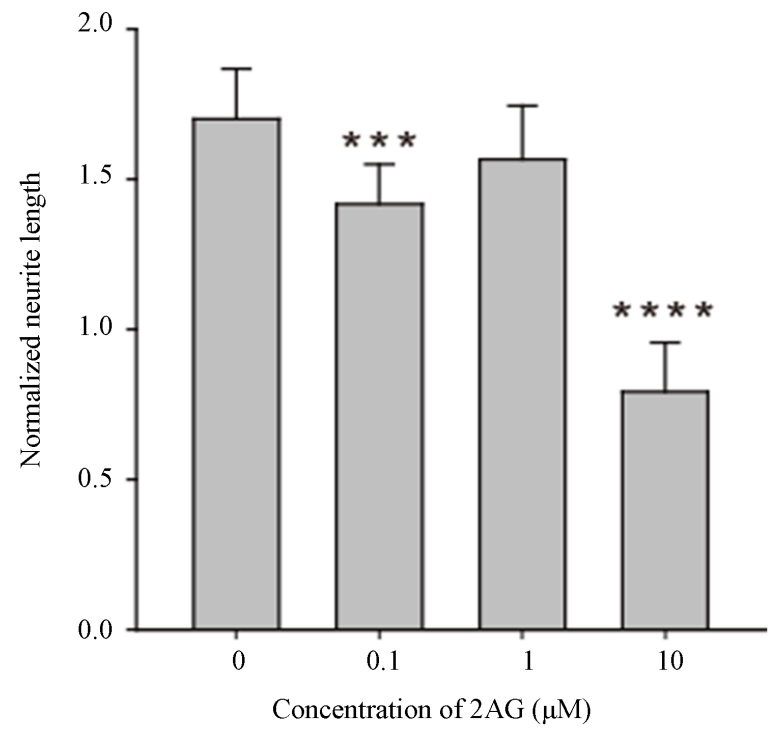

(a)

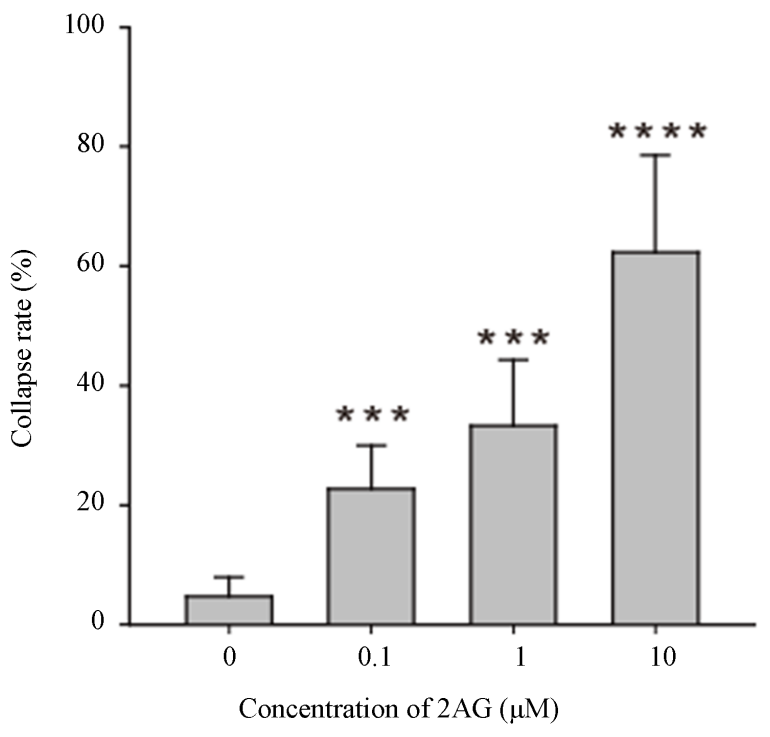

(b)

Figure 3. The effect of exposure to 2-AG for $24 \mathrm{hr}$ on neurite length (a) and the rate of growth cone collapse (b) of cultured DRG neurons. ${ }^{* * *} \mathrm{P}<0.001,{ }^{* * * *} \mathrm{P}<0.0001$, two-way ANOVA with post-hoc Bonferroni test.

elucidating the pharmacological action of cannabinoids on developing neurons. Further studies are needed to clarify the mechanisms underlying the inhibitory effect of 2-AG on DRG neurons.

We used chick explant cultures for analysis of the pharmacological actions of 2-AG because growth cones in chick explant cultures have wide fan-like shapes that are suitable for morphological analysis. The growth cone collapse assay is often used for biological analysis of endogenous factors and externally applied substances, both in developmental and regeneration studies. We previously reported that this system is applicable for pharmacological and toxicological studies of neurotropic factors, lipid mediators, and analgesic drugs [14]-[17]. However, the action of eCB may be species specific. Studies with human and other mammalian nervous tissues will be necessary for further analysis of the therapeutic use of cannabinoids.

CB1Rs are targets of exogenous cannabinoids such as cannabis. The current study suggested that activation of CB1Rs during development led to serious negative effects on neuronal growth in the peripheral nervous system. Therefore, high cannabis consumption by pregnant and lactating women may result in sensory system dysfunction in fetuses and infants, although the critical period(s) and doses of cannabis consumption in such situations 
are unclear.

The expression of CB1Rs, as well as endocannabinoid levels in the spinal cord, increased in a rat model of neuropathic pain [18] [19]. The authors speculated that eCBs function as endogenous analgesics in neuropathic pain. Clinical studies showed that cannabinoids are applicable in both acute and chronic pain patients as analgesics [20]-[22]. Because ectopic sprouting and abnormal neuronal network connections are considered a cause of neuropathic pain, the actions of eCBs on neurite extension may be crucial for inhibiting painful conditions.

\section{Conclusion}

Although the precise physiological mechanism is still unknown, our current data imply an additional action of eCBs in inhibiting formation of pain-maintenance networks. Further studies should be performed to clarify the underlying signaling cascades and morphological regulatory roles of eCBs in pain.

\section{References}

[1] Campolongo, P., Trezza, V., Ratano, P., Palmery, M. and Cuomo, V. (2011) Developmental Consequences of Perinatal Cannabis Exposure: Behavioral and Neuroendocrine Effects in Adult Rodents. Psychopharmacology, 214, 5-15. http://dx.doi.org/10.1007/s00213-010-1892-x

[2] Nutt, D.J., King, L.A. and Phillips, L.D. (2010) Drug Harms in the UK: A Multicriteria Decision Analysis. Lancet, 376, 1558-1565. http://dx.doi.org/10.1016/S0140-6736(10)61462-6

[3] Hutchings, D.E., Martin, B.R., Gamagaris, Z., Miller, N. and Fico, T. (1989) Plasma Concentrations of Delta-9-Tetrahydrocannabinol in Dams and Fetuses Following Acute or Multiple Prenatal Dosing in Rats. Life Sciences, 44, 697701. http://dx.doi.org/10.1016/0024-3205(89)90380-9

[4] Perez-Reyes, M. and Wall, M.E. (1982) Presence of Delta-9-Tetrahydrocannabinol in Human Milk. The New England Journal of Medicine, 307, 819-820. http://dx.doi.org/10.1056/NEJM198209233071311

[5] Jaques, S.C., Kingsbury, A., Henshcke, P., Chomchai, C., Clews, S., Falconer, J., Abdel-Latif, M.E., Feller, J.M. and Oei, J.L. (2014) Cannabis, the Pregnant Woman and Her Child: Weeding out the Myths. Journal of Perinatology, 34, 417-424. http://dx.doi.org/10.1038/jp.2013.180

[6] Berghuis, P., Rajnicek, A.M., Morozov, Y.M., Ross, R.A., Mulder, J., Urban, G.M., Monory, K., Marsicano, G., Matteoli, M., Canty, A., et al. (2007) Hardwiring the Brain: Endocannabinoids Shape Neuronal Connectivity. Science, 316, 1212-1216. http://dx.doi.org/10.1126/science.1137406

[7] Aguado, T., Romero, E., Monory, K., Palazuelos, J., Sendtner, M., Marsicano, G., Lutz, B., Guzman, M. and GalveRoperh, I. (2007) The CB1 Cannabinoid Receptor Mediates Excitotoxicity-Induced Neural Progenitor Proliferation and Neurogenesis. Journal of Biological Chemistry, 282, 23892-23898. http://dx.doi.org/10.1074/jbc.M700678200

[8] Harkany, T., Guzman, M., Galve-Roperh, I., Berghuis, P., Devi, L.A. and Mackie, K. (2007) The Emerging Functions of Endocannabinoid Signaling during CNS Development. Trends in Pharmacological Sciences, 28, 83-92. http://dx.doi.org/10.1016/j.tips.2006.12.004

[9] Bridges, D., Rice, A.S., Egertova, M., Elphick, M.R., Winter, J. and Michael, G.J. (2003) Localisation of Cannabinoid Receptor 1 in Rat Dorsal Root Ganglion Using in Situ Hybridisation and Immunohistochemistry. Neuroscience, 119, 803-812. http://dx.doi.org/10.1016/S0306-4522(03)00200-8

[10] Ahluwalia, J., Urban, L., Capogna, M., Bevan, S. and Nagy, I. (2000) Cannabinoid 1 Receptors Are Expressed in Nociceptive Primary Sensory Neurons. Neuroscience, 100, 685-688. http://dx.doi.org/10.1016/S0306-4522(00)00389-4

[11] Amaya, F., Shimosato, G., Kawasaki, Y., Hashimoto, S., Tanaka, Y., Ji, R.R. and Tanaka, M. (2006) Induction of CB1 Cannabinoid Receptor by Inflammation in Primary Afferent Neurons Facilitates Antihyperalgesic Effect of Peripheral CB1 Agonist. Pain, 124, 175-183. http://dx.doi.org/10.1016/j.pain.2006.04.001

[12] Senba, E. and Kashiba, H. (1996) Sensory Afferent Processing in Multi-Responsive DRG Neurons. Progress in Brain Research, 113, 387-410. http://dx.doi.org/10.1016/S0079-6123(08)61100-0

[13] Bottenstein, J.E., Skaper, S.D., Varon, S.S. and Sato, G.H. (1980) Selective Survival of Neurons from Chick Embryo Sensory Ganglionic Dissociates Utilizing Serum-Free Supplemented Medium. Experimental Cell Research, 125, 183190. http://dx.doi.org/10.1016/0014-4827(80)90202-5

[14] Radwan, I.A., Saito, S. and Gotom, F. (2002) Growth Cone Collapsing Effect of Lidocaine on DRG Neurons Is Partially Reversed by Several Neurotrophic Factors. Anesthesiology, 97, 630-635. http://dx.doi.org/10.1097/00000542-200209000-00017

[15] Saito, S., Radwan, I., Obata, H., Takahashi, K. and Goto, F. (2001) Direct Neurotoxicity of Tetracaine on Growth Cones and Neurites of Growing Neurons in Vitro. Anesthesiology, 95, 726-733. 
http://dx.doi.org/10.1097/00000542-200109000-00027

[16] Seki, T., Abdel Nazeer, A., Sekimoto, K., Guao, Y., Al-Jahdari, W. and Saito, S. (2010) Fibroblast Growth Factor and Insulin-Like Growth Factor Rescue Growth Cones of Sensory Neurites from Collapse after Tetracaine-Induced Injury. Anesthesia and Analgesia, 110, 1468-1472. http://dx.doi.org/10.1213/ANE.0b013e3181d31ea6

[17] Saito, S. (1997) Effects of Lysophosphatidic Acid on Primary Cultured Chick Neurons. Neuroscience Letters, 229, 7376. http://dx.doi.org/10.1016/S0304-3940(97)00397-2

[18] Mitrirattanakul, S., Ramakul, N., Guerrero, A.V., Matsuka, Y., Ono, T., Iwase, H., Mackie, K., Faull, K.F. and Spigelman, I. (2006) Site-Specific Increases in Peripheral Cannabinoid Receptors and Their Endogenous Ligands in a Model of Neuropathic Pain. Pain, 126, 102-114. http://dx.doi.org/10.1016/j.pain.2006.06.016

[19] Petrosino, S., Palazzo, E., de Novellis, V., Bisogno, T., Rossi, F., Maione, S. and Di Marzo, V. (2007) Changes in Spinal and Supraspinal Endocannabinoid Levels in Neuropathic Rats. Neuropharmacology, 52, 415-422. http://dx.doi.org/10.1016/j.neuropharm.2006.08.011

[20] Ashton, J.C. and Milligan, E.D. (2008) Cannabinoids for the Treatment of Neuropathic Pain: Clinical Evidence. Current Opinion in Investigational Drugs, 9, 65-75.

[21] Martin Fontelles, M.I. and Goicoechea Garcia, C. (2008) Role of Cannabinoids in the Management of Neuropathic Pain. CNS Drugs, 22, 645-653. http://dx.doi.org/10.2165/00023210-200822080-00003

[22] Hosking, R.D. and Zajicek, J.P. (2008) Therapeutic Potential of Cannabis in Pain Medicine. British Journal of Anaesthesia, 101, 59-68. http://dx.doi.org/10.1093/bja/aen119 
Scientific Research Publishing (SCIRP) is one of the largest Open Access journal publishers. It is currently publishing more than 200 open access, online, peer-reviewed journals covering a wide range of academic disciplines. SCIRP serves the worldwide academic communities and contributes to the progress and application of science with its publication.

Other selected journals from SCIRP are listed as below. Submit your manuscript to us via either submit@scirp.org or Online Submission Portal.
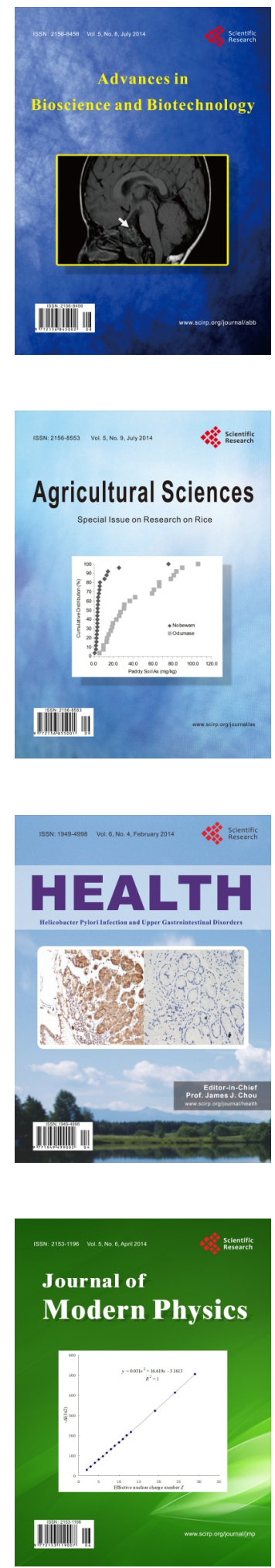
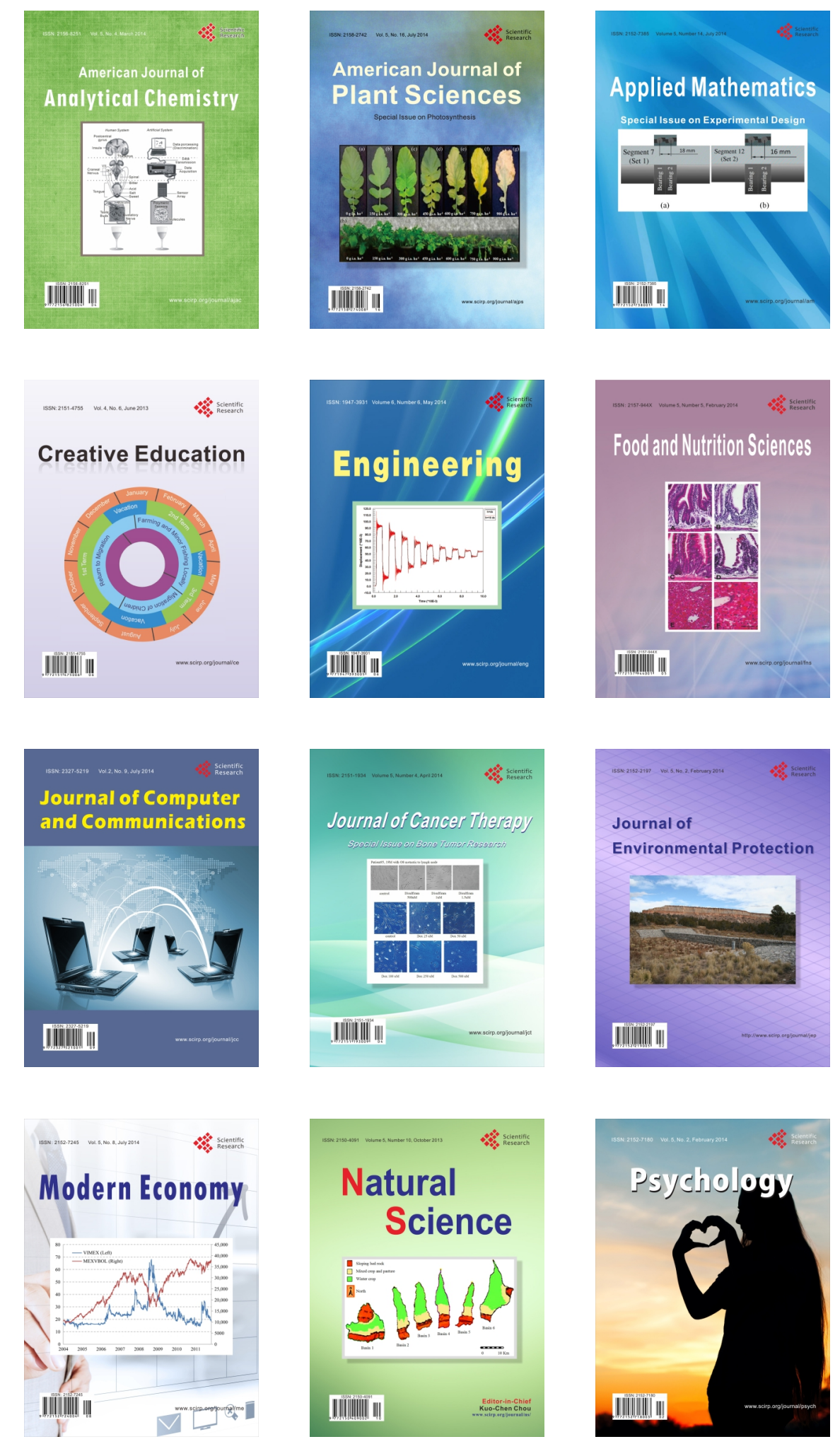